\title{
Properties of low-lying states in a diffusive quantum dot and Fock-space localization
}

\author{
Carlos Mejía-Monasterio ${ }^{1}$, Jean Richert ${ }^{2}$, Thomas Rupp ${ }^{3}$ and Hans A. Weidenmüller ${ }^{3}$ \\ ${ }^{1}$ Instituto de Fúsica, Lab. de Cuernavaca, UNAM Apdo. postal 139-B, 62191, Cuernavaca, México \\ ${ }^{2}$ Laboratoire de Physique Théorique, ULP, 3 rue de l'Université, 67084 Strasbourg, France \\ ${ }^{3}$ Max-Planck-Institut für Kernphysik, Postfach 103980, 69029 Heidelberg, Germany
}

Motivated by an experiment by Sivan et al. (Europhys. Lett. 25, 605 (1994)) and by subsequent theoretical work on localization in Fock space, we study numerically a hierarchical model for a finite many-body system of Fermions moving in a disordered potential and coupled by a two-body interaction. We focus attention on the low-lying states close to the Fermi energy. Both the spreading width and the participation number depend smoothly on excitation energy. This behavior is in keeping with naive expectations and does not display Anderson localization. We show that the model reproduces essential features of the experiment by Sivan et al.

PACS numbers: 72.15.Rn, 73.23.-b

The measurement of the quasiparticle spectrum of a diffusive quantum dot via its tunneling conductance by Sivan et al. [1] in 1994 has caused considerable theoretical activity. The experimental spectrum displayed a few narrow peaks in the vicinity of the Fermi energy, followed by a quasi-continuum [2]. The number of discrete peaks was found to be of the order of the dimensionless conductance $g$ of the dot. The results have given rise to a debate on the validity of Fermi liquid theory for diffusive quantum dots. In this theory, the low-lying excitations of a system of interacting Fermions are described as quasiparticles, i.e., free Fermions with a renormalized mass and a finite life time $\tau$, visible as peaks of width $\hbar / \tau$ in the spectrum. Are these predictions consistent with the very limited number of peaks visible in the spectrum of Ref. [1], or does disorder invalidate Fermi liquid theory?

Altshuler et al. [3] asked (and answered) this question in a precise way. These authors related the many-body problem with electron-electron interaction in a diffusive quantum dot to that of single-electron Anderson localization in real space. Many-body Fock states are introduced as Slater determinants of eigenstates of the singleparticle Hamiltonian containing kinetic energy and disorder potential. The distance in Fock space between two such states is defined as twice the minimal number of electrons which have to be moved from one singleparticle state to another in order to get from one state to the other. Matrix elements of a two-body interaction between two states differ from zero only if the distance between the two states is $\leq 4$. After introducing the Fermi energy and a particle-hole representation, the states are grouped into classes, each class being defined by the number of particle-hole $(p-h)$ excitations. The distance between classes is defined as the minimum dis- tance between any state in one class and any state in the other. In the work of Ref. [3], only couplings with distance 2 were considered. Moreover, only terms that increase the complexity of the states were kept, i.e., couplings to all states in the same and to all but one state in the next-lower class were neglected. With these assumptions, the Fock-space problem could be mapped onto a tight-binding model on the infinite Bethe lattice.

For $g \gg 1$, the existence of three regimes separated by two characteristic energies was shown. The Anderson transition [4] between localized and extended states occurs at the energy $E^{* *} \sim \Delta \sqrt{g / \ln g}$, while $E^{*} \sim \Delta \sqrt{g}$ defines an effective energy above which the many-particle states are completely mixed. Here, $\Delta$ is the average single -particle level spacing. These results were corroborated by a calculation using supersymmetry [5] and later also discussed in relation to the problem of few interacting particles in a random potential [6], to the two-body random interaction model [7], and to the level statistics of excited many-body states [8]. In Ref. [9], doubts were voiced on the claim for a delocalization transition. In Ref. [5], finite-size effects not considered in the calculation using the Bethe lattice were estimated to yield $E_{\mathrm{ch}} \sim \Delta g^{2 / 3}$ for the value of the energy at which the states become completely mixed.

The work of Refs. [3,5] uses a number of approximations which are needed to establish the connection with the Bethe lattice. In addition, it is assumed that the Bethe lattice has infinite length. These approximations are presumably valid at sufficiently high excitation energy $E$. On the other hand, the data of Ref. [1] refer to the immediate vicinity of the Fermi energy. For the interpretation of these data, it is important to know whether the results of the high-energy approximations apply. For instance, at low excitation energy and with $\varepsilon=E / \Delta$, the number of accessible classes has a strong cutoff $\sim \sqrt{\varepsilon}$.

To answer this question, we investigate in this Letter the low-lying states of the many-body problem with disorder and interaction in the framework of a model which is more realistic than but retains the spirit of Refs. [3,5]. We avoid the approximations made in these papers which are needed to obtain the structure of the Bethe lattice. We pay the price that we cannot use analytical approximations and must rely on numerical simulation.

The Hamiltonian for spinless electrons (Fermions) has the form $H=H_{0}+V$, with $H_{0}=\sum_{k} \epsilon_{k} a_{k}^{\dagger} a_{k}$ the unperturbed Hamiltonian, a sum of single-particle operators, and $V$ the two-body interaction. The symbol $\epsilon_{k}$ 
denotes the single particle eigenvalues, and $a_{k}^{\dagger}$ generates the single particle states $|k\rangle$. The single-particle Hamiltonian contains a random potential. Therefore, the states $|k\rangle$ and energies $\epsilon_{k}$ have stochastic properties. In an energy interval of length $g \Delta$, these properties coincide with those of the Gaussian orthogonal ensemble (GOE) of random matrices. We use the classification scheme defined above and consider classes $m$ of Fock states with $m$ particles and $(m-1)$ holes. Here, $m=1,2, \ldots$ We denote the states by $|m, i\rangle$, where $i$ is an index running over the states of the class. The associated energies, given by sums of the $\epsilon_{k}$ 's, are denoted by $E_{m, i}$, so that $H_{0}|m, i\rangle=E_{m, i}|m, i\rangle$. To implement this model, we take the energies $\epsilon_{k}$ after unfolding from the center of the Wigner semicircle for the GOE. We choose the Fermi energy equal to zero.

The unperturbed mean level density $\rho_{m}^{0}(\varepsilon)$ in class $m$ is given by $[5] \rho_{m}^{0}(\varepsilon)=\Delta^{-1} \varepsilon^{2 m} /[(m+1) ! m !(2 m) !]$, where $\varepsilon=E / \Delta$ and $E$ is the excitation energy. With $\varepsilon$ fixed, $\rho_{m}^{0}$ grows strongly with $m$ until it suddenly drops to almost zero at $m \sim \sqrt{\varepsilon}$. Hence, at any value of $\varepsilon$ only a limited number of classes contributes to the total level density of the unperturbed system $(V=0)$. In the vicinity of the Fermi energy, this number is one or two. This fact is important for the experiment of Ref. [1]. Indeed, if the localization length is larger than two, then localization in Fock space can have no bearing on the spectrum in the vicinity of the Fermi surface.

The interaction operator $V$ mixes the states $|m, i\rangle$. We suppose that the diagonal part of the interaction is included in $H_{0}$ by use of the Hartree-Fock method, without affecting the statistical properties of either the energies $E_{m, i}$ or of the states $|m, i\rangle$. The matrix elements of $V$ between two different Fock states $|m, i\rangle$ and $|n, j\rangle$ vanish unless $|m-n|=0$ or 1 and unless the Fock distance of both states is $\leq 4$. (The case $|m-n|=2$ requires creation or destruction of two particle-hole pairs out of the Fermi sea. Such processes contribute to the unlinked diagrams of perturbation theory and are not considered here. Calculations including couplings with $|m-n|=2$ yielded results which did not differ significantly.) The non-vanishing matrix elements of $V$ are assumed $[3,10]$ to have a Gaussian distribution centered at zero with variance $\overline{V^{2}}=(\Delta / g)^{2}$. The mixing of the states $|m, i\rangle$ depends on their spacing and on the strength of $V$, i.e., on the value of $g$. In our model, the strength of $V$ does not depend on excitation energy. This fact will cause stronger mixing of the higher-lying states with smaller spacings. The effect is compensated because out of the larger number of states, a decreasing fraction couples to a given one. We allow for couplings between states in the same class. This introduces terms which are explicitly excluded in the case of the Bethe lattice.

In our numerical work, the energy scale is defined by $\Delta$. Our only parameter is the dimensionless conductance $g$. The dimension of the matrices was limited by a cutoff $E_{\text {cut off }} \gg \Delta$ : All Fock states with energies $E_{m, i} \geq E_{\text {cut off }}$ were omitted. For each realization of $H, E_{\text {cut off }}$ was cho- sen in such a way that the matrix dimension was 1000 . This corresponds roughly to $E_{\text {cutoff }} \sim 18 \Delta$. Since our single-particle energies are drawn from the center of a GOE distribution, consistency requires that the Thouless energy $g \Delta$ is at least as large as the energy interval considered. The eigenstates $|\alpha\rangle$ and eigenvalues $E_{\alpha}$ of the full problem were obtained by diagonalization. We have checked that the results presented below are independent of $E_{\text {cut off }}$. We now present two statistical measures suitable for a test of localization in Fock space.

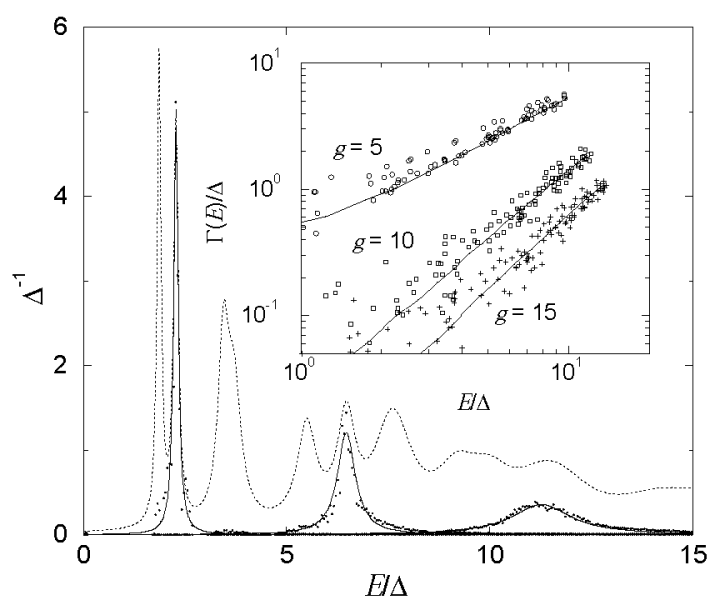

FIG. 1. Quasiparticle line shapes $\rho_{1, i}$ for the $1 p-0 h$ states $|1,2\rangle,|1,6\rangle$, and $|1,11\rangle$ (full curves). The dotted curve is the quasiparticle spectrum of all $1 p-0 h$ states. The inset shows the spreading width $\Gamma$ versus $E$ for $g=5$ (circles), $g=10$ (squares), and $g=15$ (crosses) together with the Golden Rule predictions $\Gamma_{\mathrm{GR}}(E)$ (full curves).

Quasiparticle spectrum. Adding an electron to a quantum dot with a filled Fermi sea creates a $1 p-0 h$ Fock state $|1, i\rangle$. The interaction $V$ spreads this state over a set of eigenstates $|\alpha\rangle$. The spectral shape of the resulting quasiparticle peak, also referred to as the local density of states, is given by

$$
\rho_{1, i}(E)=\left\langle\sum_{\alpha}|\langle\alpha \mid 1, i\rangle|^{2} \delta\left(E-E_{\alpha}\right)\right\rangle .
$$

The brackets denote the ensemble average. In order to obtain meaningful plots, we have used only a single realization of the single-particle energies but 100 realizations of the interaction matrix elements. To reduce the density of the resulting data set, we have averaged our results over small energy windows. This yields the dots shown in Fig. 1 for three selected $1 p-0 h$ states at $g=10$. The fits with the Lorentzian

$$
\frac{1}{2 \pi} \frac{\Gamma_{1, i}}{\left(E-\epsilon_{1, i}\right)^{2}+\Gamma_{1, i}^{2} / 4}
$$

(full curves) fix the centers $\epsilon_{1, i}$ of the quasiparticle peaks and the spreading widths $\Gamma_{1, i}$. Adding the Lorentzian 
line shapes of all $1 p-0 h$ states yields the quasiparticle spectrum shown as dotted line.

Spreading width. Plotting $\Gamma_{1, i}$ for all states $|1, i\rangle$ versus the state energies $\epsilon_{1, i}$, we obtain the spreading width $\Gamma(E)$ as a function of excitation energy. For several values of $g$, this dependence is displayed in the inset of Fig. 1. We compare our results with the prediction of the Golden Rule. We study the mixing of $1 p-0 h$ states. Each one of these is directly coupled to both all other $1 p-0 h$ states and all $2 p-1 h$ states. The $1 p-0 h$ states have mean spacing $\Delta$. At the coupling strengths $(g \geq 5)$ shown in Fig. 1, these states are barely mixed with each other. Therefore we keep only the density of $2 p-1 h$ states, $\rho_{2}(E)$, as the appropriate quantity to use in the Golden Rule expression

$$
\Gamma_{\mathrm{GR}}(E)=2 \pi(\Delta / g)^{2} \rho_{2}(E) .
$$

We point out that this relation is valid beyond perturbation theory if the density of final states is taken to be the exact (rather than the unperturbed) level density. The exact density in class $m$ is defined as

$$
\rho_{m}(E)=\left\langle\sum_{i, \alpha}|\langle\alpha \mid m, i\rangle|^{2} \delta\left(E-E_{\alpha}\right)\right\rangle .
$$

The brackets again denote the ensemble average which in practice we perform over 100 realizations of the full Hamiltonian $H$. The three full lines in the inset of Fig. 1 show $\Gamma_{\mathrm{GR}}(E)$ for the three values of $g$. With decreasing $g$, i.e., increasing interaction $V, \Gamma_{G R}(E)$ increases while the slope of $\Gamma_{G R}(E)$ decreases. These facts reflect both the $1 / g^{2}$ dependence of $\overline{V^{2}}$ and the behavior of $\rho_{2}(E)$. For $g=10$, we show in Fig. $2 \rho_{1}(E)$, $\rho_{2}(E), \rho_{t}(E)=\sum_{m} \rho_{m}(E)$, and the unperturbed density of states $\rho_{2}^{0}(E)$. The latter differs substantially from $\rho_{2}(E)$ : The interaction causes the density to become wider, thereby reducing its slope. This accounts for the behavior of $\Gamma_{\mathrm{GR}}(E)$ in the inset of Fig. 1. In Refs. [3,5], it was emphasized that localization in Fock space invalidates the Golden Rule. The quantitative agreement between our numerical results and the expression (3) down to very small energies shown in Fig. 1 is, thus, a very strong argument against the occurrence of localization in the energy range investigated in this paper. The weak mixing of the low-lying states is entirely explained by the low density of states right above the Fermi energy and is not due to additional restrictions in Fock space.

Qualitative features of the quasiparticle spectrum in Fig. 1 are in good agreement with the results of the experiment by Sivan et al. $[1,2]$ : A few nearly discrete quasiparticle peaks with $\Gamma<\Delta$ occur right above the Fermi energy. The width $\Gamma$ grows with excitation energy until $\Gamma \approx \Delta$ at $E \approx g \Delta$ where the spectrum becomes quasi-continuous. For an accurate simulation of Sivan's experiment, however, the $1 p-0 h$ excitations should be built upon the ground state of interacting particles rather than upon the filled Fermi sea. Calculations using such an interacting ground state reproduced the results shown in Figs. 1 and 2, except for $g=5$. Here the spreading widths were at the upper end of the statistical fluctuations shown in Fig. 1, while the Golden Rule underestimates $\Gamma$ by about a factor two. The clear hierarchical structure needed to study localization is lost when we start from an interacting ground state. Therefore, we kept the simple model outlined above.

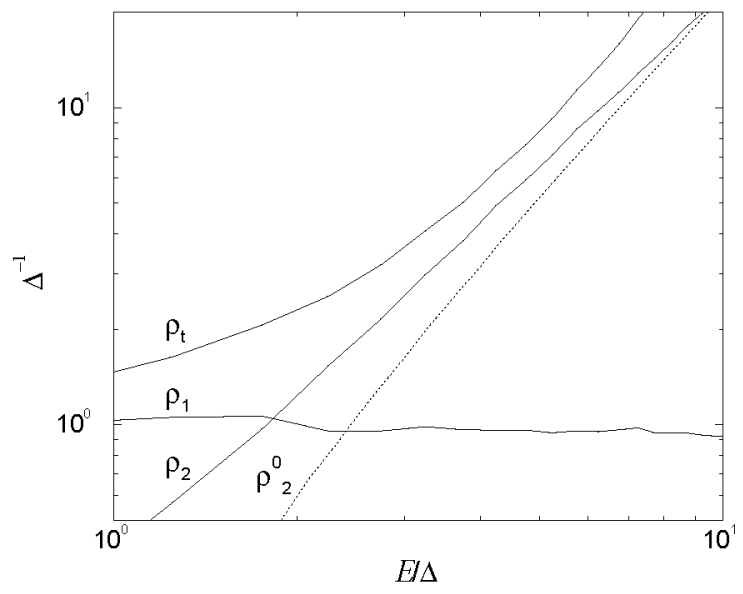

FIG. 2. Densities $\rho_{1}(E), \rho_{2}(E)$, and $\rho_{t}(E)$ for $g=10$ as defined in the text. For comparison we also show $\rho_{2}^{0}(E)$.

Participation number. The average participation number often serves as a measure of localization. The participation number for the Fock state $|m, i\rangle$ is defined by

$$
R_{m, i}=\left(\sum_{\alpha}|\langle\alpha \mid m, i\rangle|^{4}\right)^{-1}
$$

If the state $|m, i\rangle$ is localized, $R_{m, i} \approx 1$, while $R_{m, i}$ increases monotonically with increasing mixing. In the thermodynamic limit, $R_{m, i}$ is unbounded. In the case of finite matrix dimension $N$ and the GOE, there exists an upper bound $R_{\max }=(1 / 3) N$ for $R_{m, i}$. In Fig. 3 we show the average participation number $R_{1}(E)$ of class 1. This quantity is obtained by plotting the values of $R_{1, i}$ for all available $i$ versus the corresponding energies $E_{1, i}$ for a number of realizations and averaging the result over energy. For weak interaction (here $g=15$ ) and low excitation energies, the Fock states $|1, i\rangle$ are localized because the spreading widths of the quasiparticle states are small compared to the mean level spacing. With increasing $V(g=5)$ the mixing becomes larger even at low excitation energies. We only find smooth transitions to mixed states, both with increasing excitation energy $E$ and with increasing interaction $V$. From the absence of any discontinuity in $R_{1}(E)$, we conclude that there is no evidence for a localization transition in Fock space. For $g=15$, the delocalization thresholds predicted in Refs. $[3,5]$ have the values $E^{* *} \approx 2.34 \Delta$ and $E^{*} \approx 3.87 \Delta$. We find $R_{1}(E) \lesssim 2$ for $E \leq 5$ which shows that at these 
energies, the mixing of Fock states is rather weak. More generally, an estimate of the number of eigenstates $|\alpha\rangle$ contributing to a Fock state $|1, i\rangle$ at energy $E$ and, thus, of the average participation number $R_{1}(E)$ is given by $R_{1}^{\mathrm{GR}}(E)=\Gamma_{\mathrm{GR}}(E) \rho_{t}(E)$ (see Eq. (3) and Fig. 2). We find this relation to be correct for sufficiently high energies $E$ (see Fig. 3) while $R_{1}^{G R}(E)$ overestimates $R_{1}(E)$ at low energies since by definition, $R_{1}(E) \geq 1$ whereas the level density becomes very small. For a localization transition, we would expect $R_{1}(E)$ to lie below the Golden Rule estimate. This is not the case.

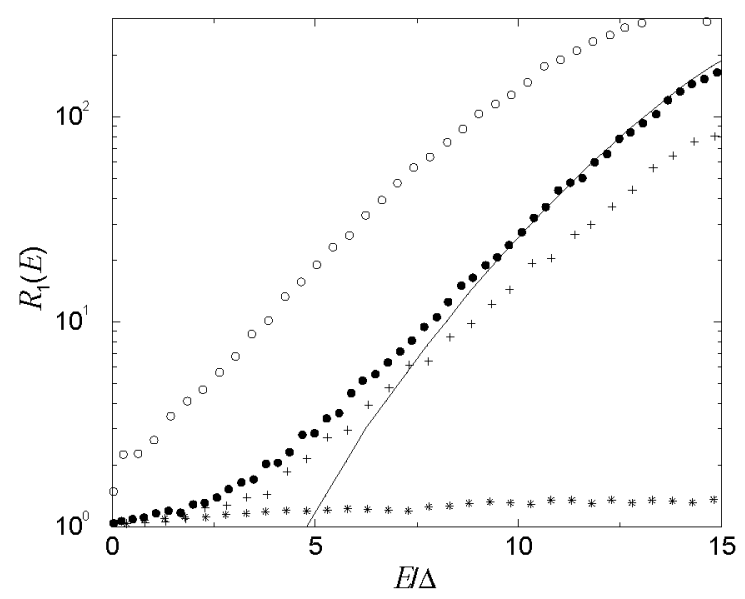

FIG. 3. Participation number $R_{1}(E)$ of the $1 p-0 h$ states for $g=15$ (full circles) and $g=5$ (empty circles). The estimate $R_{1}^{\mathrm{GR}}(E)$ for $g=15$ is plotted as a full curve. In addition the results for applying a loopless Bethe lattice model (crosses) and considering couplings over Fock distance 2 only (stars) are shown (both $g=15$ ).

Sensitivity to coupling scheme. How do our results change with coupling scheme, i.e., with the omission of those interaction terms which spoil the analogy with the Bethe lattice? In the coupling scheme applied so far we allowed for couplings between states of Fock distances 2 or 4 , in keeping with the assumption of a two-body interaction. The stars in Fig. 3 show the average participation number for $1 p-0 h$ Fock states when only couplings between states of Fock distance 2 are taken into account. This restriction means that only one particle is allowed to change its single-particle state in an interaction and no other particle can compensate the energy difference implied by this transition. In effect couplings between states close in energy are neglected.

In order to test the Bethe lattice assumption, i.e., a Fock space topology without any loops, we first assumed that every Fock state $|m, i\rangle$ with $m \geq 2$ is coupled to only one state in class $m-1$. This state was taken to be the one closest in energy to $|m, i\rangle$. Couplings within each class were neglected. Couplings with Fock-space distance 4 were taken into account. This model yielded a set of disconnected trees each starting from one $1 p-0 h$ state. As each Fock state belongs only to exactly one tree, the trees were all of rather small size. The resulting participation numbers were much smaller than those of the full calculation, especially at high energies. This is unsatisfactory. We then considered a second model. We coupled each $1 p-0 h$ state to all $2 p-1 h$ states and kept the couplings between higher classes as previously. Now all Fock states except the $1 p-0 h$ states participate in each tree. The resulting average participation numbers (crosses in Fig. 3) agree rather well with the full calculation.

In conclusion, we presented a numerical study of the eigenvalues and eigenfunctions of a finite Fermionic many-body problem with random single-particle energies and a random two-body interaction. Our model avoids the simplifications of the Bethe lattice. Matrix diagonalization with a cutoff yielded results restricted to the vicinity of the Fermi surface and insensitive to the choice of the cutoff. We found that it is important to keep couplings between states with a distance 4 in Fock space. Moreover, we showed that for sensible values of the conductance $g$, our model is able to reproduce essential features of the experiment of Ref. [1]. Calculated values of the spreading width and the participation number indicate some degree of localization, depending on interaction strength and excitation energy. However, in contrast to analytical predictions based on a high-energy approximation and on the Bethe lattice (which claim a twofold localization transition) as well as those of Ref. [6], we have only found a smooth transition from almost localized to delocalized states. This transition can be fully understood in terms of the density of available $1 p-0 h$ and $2 p-1 h$ states and does not display Anderson localization. Our results show that the behavior of a finite Fermi system at low excitation energy and at zero temperature differs profoundly from the thermodynamic limit.

Acknowledgements. We thank Y. Imry, T. Seligman, R. Jalabert and T. Wilke for stimulating discussions.

[1] U. Sivan, F. P. Milliken, K. Milkove, S. Rishton, Y. Lee, J. M. Hong, V. Boegli, D. Kern and M. DeFranza, Europhys. Lett. 25, 605 (1994).

[2] U. Sivan, Y. Imry and A. G. Aronov, Europhys. Lett. 28, 115 (1994).

[3] B. L. Altshuler, Y. Gefen, A. Kamenev and L. S. Levitov, Phys. Rev. Lett. 78, 2803 (1997).

[4] R. Abou-Chacra, P. W. Anderson and D. J. Thouless, J. Phys. C6, 1734 (1973).

[5] A. Mirlin and Y. Fyodorov, Phys. Rev. B 56, 13393 (1997).

[6] D. Weinmann, J.-L. Pichard and Y. Imry, J. Phys. I France 7, 1559 (1997).

[7] Ph. Jacquod and D. L. Shepelyansky, Phys. Rev. Lett. 79, 1837 (1997).

[8] R. Berkovits and Y. Avishai, Phys. Rev. Lett. 80, 568 (1998).

[9] P. G. Silvestrov, Phys. Rev. Lett. 79, 3994 (1997).

[10] Ya. M. Blanter, Phys. Rev. B 54, 12807 (1996). 